\title{
EDITORIAL
}

\section{Health effects of coarse particles in ambient air: messages for research and decision-making}

\author{
T. Sandström*, D. Nowak ${ }^{\#}$ and L. van Bree
}

I $\mathrm{n}$ the current issue of European Respiratory Journal, BRUNEKREEF and FORSBERG [1] present a review article based on a systematical appraisal of the existing literature on health effects of fine and coarse particulate air pollution. Even though systematical searches may often be assumed as a foundation for review papers, this is not always performed. Herein lies the strength of the present article, and most importantly, the authors come to different conclusions than earlier paradigms when all available literature is taken into account.

Adverse health effects of ambient air pollution in general, and particulate matter (PM) in particular, have drawn considerable attention over recent years [2, 3]. Health effects range from increases in respiratory symptoms and increased rescue medication use, to asthma and chronic obstructive pulmonary disease deteriorations resulting in emergency room treatments, and even reduced lung growth in children [4]. Additionally, considerable attention has been focused on increased mortality in respiratory and cardiovascular conditions. Estimates, although uncertain, have suggested an equal or exceeded number of premature deaths per year, as compared with traffic accidents [5].

Quantitative health impact assessment (HIA), in general, including that for air pollution, represents a new approach to the evaluation of the current state-of-the-environment and of future conditions following specific abatement scenarios. The importance of HIA is strongly endorsed by governments and it is recommended to quantitatively assess adverse health impacts and possible inequalities in the population. Furthermore, the importance of HIA has been recognised through Article 152 of the Amsterdam Treaty calling for the European Union to examine the possible impact of major policies on health. However, to run a HIA for ambient air particles is extremely complex and has been suffering from a considerable lack of knowledge on the following: 1) particle composition; 2) mechanistic toxicological insight in particle components causally responsible for cardio-respiratory health effects; 3) airway deposition data for various particle size fractions; and 4) epidemiological evidence for relationships of various ambient air particle components with observed health effects in the pollution.

*Dept of Respiratory Medicine and Allergy, University Hospital, Umeå, Sweden. \#Institut und Poliklinik für Arbeits - und Umweltmedizin, Der Ludwig - Maximilians - Universität, Munich, Germany. "Netherlands Environmental Assessment Agency, Bilthoven, The Netherlands.

CORRESPONDENCE: T. Sandström, Dept of Respiratory Medicine and Allergy, University Hospital Umeå, Sweden. Fax: 46 90141369. E-mail: thomas.sandstrom@lung.umu.se
Therefore, policy makers, as in the current Clean Air for Europe (CAFE) programme of the European Commission, are struggling with the question of how to decide on health- and cost-effective air pollution policy, air quality standards and target emission control. A framework of air quality guidelines (World Health Organization, Europe) and air quality standards and emission ceilings (European Union, Member States) have been set in place to improve poor air quality and to reduce the major health impacts. For ambient particles this control strategy is focussing on PM10 or PM2.5, being particles with a diameter smaller than 10 and $2.5 \mu \mathrm{m}$, respectively. For both particle fractions it is believed that a safe concentration (a "no-effect level or threshold") is unlikely to exist. Consequently, compliance with a standard can reduce the human health impact to a certain extent, but cannot prevent it. Even if the current ambient air quality targets for particles, set for 2005 and 2010, are met, considerable health impacts are still likely. So, there is a considerable responsibility for the health effects research community to produce clear views on mechanisms and causative PM fractions, which would, hopefully, allow decision-makers to better focus their abatement policy in an effective way.

In recent years, a range of studies have tried to disentangle the complex PM issues. First, it seems that PM originates partly from natural sources (wind-born soil, sea-spray and organic compounds) and from man-made activities (combustion of fossil fuels, industry, vehicle and road wear) and may, therefore, have a heterogeneous composition depending on weather conditions, type and strengths of sources, and exposure location. PM can range in particle size from a few $\mathrm{nm}$ to tens of $\mu \mathrm{m}$. Secondly, different particle size fractions of PM, i.e. PM2.5-10 (coarse particles; their size ranges from 2.5$10 \mu \mathrm{m}$ ), PM2.5 (fine particles; their size ranges from $0.1-2.5 \mu \mathrm{m}$ ) and PM0.1 (ultrafine particles; their size is $<0.1 \mu \mathrm{m}$ ), are suggested to deposit differently along the respiratory system with increased deposition in subjects with airway obstruction. Thirdly, the toxicological database on the type of particle sizes and chemical compositions that favour toxicity is rapidly growing. This is in part due to recent European Union support for large projects in the air pollution arena. Importantly, coarse and fine PM fractions both seem capable of inducing toxicity, and at least partly through oxidative stress pathways [6]. It is also suggested that primary, carbonaceous PM components may be more important for health effects than the secondary components, such as sulphates and nitrates.

Toxicological studies in humans and animals suggest that PM may adversely affect the function of the respiratory system, the blood vessels and the heart [7]. The results do, for obvious 
reasons, arise from studies using experimental conditions, which gives some uncertainties when directly extrapolating these data to the actual human ambient exposure conditions. Controlled inhalation of PM at concentrations well above ambient levels results in exacerbations of symptoms in patients with (mild or moderate) pre-existing lung disease, such as asthma, as well as heart and blood vessel disease. Toxicological studies suggest that these effects are due to induction of lung inflammation, disturbances in heart rhythm, alterations in blood viscosity and oxygen deprivation. Remarkably, PM airway deposition models suggest that these types of mild/ moderate patients receive much higher doses in their airways and lungs compared with healthy individuals. It could, therefore, be that the more severe patients receive even higher doses and/or their tissue responds more extensively to a certain dose. All these effects may partly explain why these people are at increased risks from PM. Analogous effects, albeit at much lower intensity, have also been observed in healthy individuals.

The toxicological and epidemiological research communities have invested considerable effort into unraveling the complex PM issues and, although the results on many aspects are good, much information is still lacking. Furthermore, considerable amounts of information that can be made available, have not always been sufficiently compiled and critically analysed. In the article by BRUNEKREEF and FORSBERG [1], the authors have systematically evaluated the existing data on health effects and PM2.5-10 and PM2.5. The conclusion of the article shows that there is evidence to suggest that both these PM fractions are able to elicit adverse health effects. Differences seen in different studies may be, in part, due to variations in sources and chemical composition of the particles of the different size ranges. The coarse PM2.5-10 fraction may play an important role for certain aspects of morbidity, and may also contribute to mortality, although possibly somewhat less than the finer fraction PM2.5. The article brings an important message, both for science and for the future and, hopefully, better targeted air pollution control policy. Major steps forward in PM health risk assessment, standard setting, and emission control are to gain insight into the major health-relevant particle features with respect to particle size (coarse, fine and ultrafine) and composition (primary and secondary fractions), and to link these to responsible emissions and sources. The article by BRUNKREEF and FORSBERG [1] voices substantial adverse health effects by the coarse particles in the PM2.5-10 fraction and shows the evidence needed for diversification of the current PM10 health risk assessment.

The conclusion of this study offers evidence to treat the coarse particle fraction differently from the fine fraction and provides European Union CAFE decision-makers with information to expand the particulate matter risk assessment dossier, focusing at present almost exclusively on PM2.5, and leaving particulate matter 10 or particulate matter $2.5-10$ regrettably unaltered. The outcome of this study should, therefore, be brought to the immediate attention of the European Committee Clean Air for Europe programme in order to maximise its impact on decision-making for Europe. Meanwhile, clinical scientists, toxicologists and epidemiologists need to enhance their efforts to disentangle the complex mechanisms whereby chemical and physiological properties of air pollution particles produce adverse respiratory and cardiovascular effects.

\section{REFERENCES}

1 Brunekreef B, Forsberg B. Epidemiological evidence of effects of coarse airbourne particles on health. Eur Respir J 2005; 26: 309-318.

2 Dockery DW, Pope AC, Xu X, et al. An association between air pollution and mortality in six U.S. cities. N Engl J Med 1993; 329: 1753-1759.

3 Brunekreef B, Holgate ST. Air pollution and health. Lancet 2002; 360: 1233-1242.

4 Gauderman WJ, Avol E, Gilliland F, et al. The effect of air pollution on lung development from 10 to 18 years of age. N Engl J Med 2004; 351: 1057-1067.

5 Künzli N, Kaiser R, Medina S, et al. Public-health impact of outdoor and traffic-related air pollution: a European assessment. Lancet 2000; 356: 795-801.

6 Kelly FJ, Sandstrom T. Air pollution, oxidative stress, and allergic response. Lancet 2004; 363: 95-96.

7 Stenfors N, Nordenhall C, Salvi SS, et al. Different airway inflammatory responses in asthmatic and healthy humans exposed to diesel. Eur Respir J 2004; 23: 82-86. 